\title{
Towards a Sustainable Low-Carbon Rural Society: Case Humppila
}

Winfried Schäfer ${ }^{1)}$, Taija Sinkko'), Rabbe Thun ${ }^{3)}$

${ }^{1)}$ MTT, Vakolantie 55, 03400 Vihti, winfried.schafer@mtt.fi

${ }^{2)}$ MTT, 00014 Helsingin yliopisto, taija.sinkko@mtt.fi

${ }^{3)}$ MTT, ET-talo, 31600 Jokioinen, rabbe.thun@mtt.fi

\section{Introduction}

Many rural municipalities aim energy self-sufficiency using renewable energy sources on regional level due to rising energy prices, reliability of steady supply and environmental pollution. A new draft directive of the EU in January 2008 triggers the efforts to raise the proportion of renewable sources in energy production by $20 \%$ until 2020. This means that Finland should rise the proportion of renewable energy resources in energy production from about $28.5 \%$ in 2005 to about $38.5 \%$ until 2020 (Heinimö 2008) The objective of this paper is to find whether an example rural municipality can achieve this goal and whether there is the potential to become energy self-sufficient and to close material flows.

\section{Methodology}

We employed three methods: embodied energy assessment, life cycle analysis (LCA), and emergy accounting.

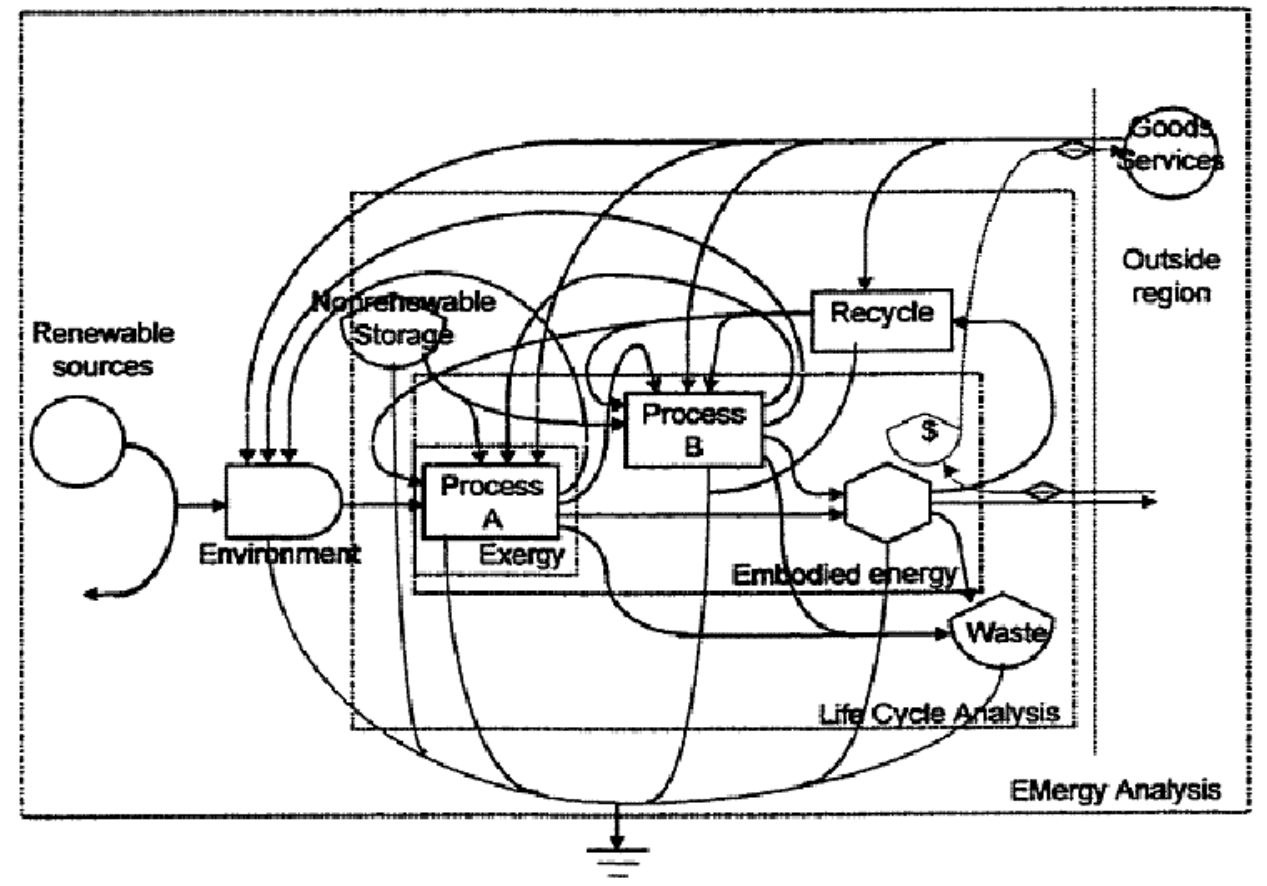

Figure 1: Scale and scope of various methods evaluating material and energy requirements of processes. Each dashed rectangle encloses the proportions of systems evaluated with each method (Buranakarn 1998).

Embedded energy assessment includes direct and indirect energy inputs of processes. The energy surplus from solar radiation conversion into all kinds of energy inputs per capita and square meter of the surface area is a measure for sustainability of renewable energy production (Schäfer 2008).

LCA (SFS-EN ISO 14040) was initially developed for environmental assessment of industrial production and includes only direct environmental inputs. The method does not distinguish different energy qualities. Usually labour input is not included.

Emergy accounting (Odum 1996) is a holistic energy evaluation method rooted in thermodynamics and systems thinking increasingly used worldwide that accounts for both natural and economic resources used up within ecosystems (Bastianoni et al. 2009, Franzese et al. 2009, Paoli et al. 2008, Pulselli, et al. 2008, Siracusa et al. 2008, Jiang et al. 2007, Rydberg et al. 2006). Different forms of energy, materials, human labour and economic services are all evaluated on a common basis 
by converting them into equivalents of the solar energy expressed as solar equivalent Joule (sej). The conversion factor is called transformity. Odum 1996 describes ten different methods to calculate transformity.

\section{Results and discussion}

A preliminary LCA analysis showed, that Humppila could produce $75 \%$ of the electricity and $90 \%$ of the heat consumption utilising the local biomass resources in two CHP plants and recycling residues from anaerobic digestion to substitute chemical fertilisers. Results also indicated that greenhouse gas emissions of Humppila could decrease by about 6000 tonnes of carbon dioxide equivalents per year (Sinkko, T. 2009).

The solar-energy balance (Schäfer 2008 shows that the municipality receives yearly 4.2E+17 J renewable solar energy assuming a mean albedo of $14.3 \%$. The energy consumption by oil-derived products is $2.6 \mathrm{E}+14 \mathrm{~J}$ and the electric power consumption $8.1 \mathrm{E}+13 \mathrm{~J}$. Thus, an overall solar energy conversion efficiency of $0.8 \%$ would make Humppila completely independent from non-renewable imported energy sources. The heat value of the biomass potential on the area of Humppila is 8.2E+14 J (29.9\% wood (Sinkko 2009), 70.1\% crops) corresponding to a solar energy conversion efficiency of $2.4 \%$ ( $1.3 \%$ wood, $3.5 \%$ crop). In contrast to Finland, Humppila could easily cover its energy consumption by renewable solar energy techniques.

Table 1: Emergy evaluation of the Humppila municipality. R: local renewable resources, $\mathrm{N}$ : local not renewable resources, F: imported energy, G: purchased goods, S: services, E: Exports. Emergy = raw amount * transformity (Odum 1996).

\begin{tabular}{|c|c|c|c|c|c|c|}
\hline Note & Input/output & & Raw amount & unit & $\begin{array}{r}\text { Transformity } \\
\text { sej/unit }\end{array}$ & $\begin{array}{r}\text { Emergy } \\
\text { E+17 sej/year }\end{array}$ \\
\hline \multicolumn{7}{|c|}{ Renewable resources } \\
\hline 1 & | sunlight & $\mathrm{R}$ & $4.22 \mathrm{E}+17$ & $\mathrm{~J} /$ year & 1 & 4.22 \\
\hline 2 & Wind, kinetic energy & & $1.29 \mathrm{E}+13$ & $\mathrm{~J} /$ year & $2.51 \mathrm{E}+03$ & 0.32 \\
\hline 3 & Rain, evapotranspired & & $2.89 \mathrm{E}+12$ & $\mathrm{~J} /$ year & $3.05 \mathrm{E}+04$ & 0.88 \\
\hline \multicolumn{7}{|c|}{ Internal transformations } \\
\hline 4 & Crop production & & $5.77 \mathrm{E}+14$ & $\mathrm{~J} /$ year & $3.31 \mathrm{E}+04$ & 191.11 \\
\hline 5 & Livestock production & & $5.34 \mathrm{E}+13$ & $\mathrm{~J} /$ year & $2.58 \mathrm{E}+05$ & 137.73 \\
\hline 6 & Forest extraction & & $1.47 \mathrm{E}+14$ & $\mathrm{~J} /$ year & $5.04 \mathrm{E}+04$ & 74.31 \\
\hline 7 & District Heating & & $4.68 \mathrm{E}+13$ & $\mathrm{~J} /$ year & $3.32 \mathrm{E}+04$ & 15.56 \\
\hline \multicolumn{7}{|c|}{ Non-renewable resources from within system } \\
\hline 8 & | Top soil & $\mathrm{N}$ & $6.35 \mathrm{E}+12$ & $\mathrm{~J} /$ year & $1.24 \mathrm{E}+05$ & 7.88 \\
\hline \multicolumn{7}{|c|}{ Imports and outside sources } \\
\hline 9 & Oil derived products & $\mathrm{F}$ & $2.63 \mathrm{E}+14$ & $\mathrm{~J} /$ year & $1.11 \mathrm{E}+05$ & 291.86 \\
\hline 10 & Electric power & $\mathrm{F}$ & $8.14 \mathrm{E}+13$ & $\mathrm{~J} /$ year & $2.51 \mathrm{E}+05$ & 204.21 \\
\hline 11 & Metals & $\mathrm{G}$ & $2.00 \mathrm{E}+07$ & g/year & $6.28 \mathrm{E}+09$ & 1.26 \\
\hline 12 & Nitrogen & $\mathrm{G}$ & $4.73 \mathrm{E}+08$ & g/year & $7.71 \mathrm{E}+09$ & 36.45 \\
\hline 13 & Phosphate & $\mathrm{G}$ & $5.91 \mathrm{E}+07$ & g/year & $2.98 \mathrm{E}+10$ & 17.61 \\
\hline 14 & Potash & $\mathrm{G}$ & $1.65 \mathrm{E}+08$ & g/year & $2.92 \mathrm{E}+09$ & 4.83 \\
\hline 15 & Pesticides \& Herbicides & $\mathrm{G}$ & $1.65 \mathrm{E}+07$ & g/year & $2.48 \mathrm{E}+10$ & 4.10 \\
\hline 16 & Feed & $\mathrm{G}$ & $1.58 \mathrm{E}+06$ & $€ /$ year & $3.39 \mathrm{E}+12$ & 53.53 \\
\hline 17 & Vehicles & $\mathrm{G}$ & $3.33 \mathrm{E}+08$ & $\mathrm{~g} /$ year & $6.70 \mathrm{E}+09$ & 22.32 \\
\hline 18 & Service and food imports & $\mathrm{S}$ & $1.43 \mathrm{E}+07$ & $€ /$ year & $3.39 \mathrm{E}+12$ & 485.65 \\
\hline \multicolumn{7}{|l|}{ Exports } \\
\hline 19 & Crop products & $\mathrm{E}$ & $2.43 \mathrm{E}+14$ & $\mathrm{~J} /$ year & $1.59 \mathrm{E}+05$ & 386.88 \\
\hline 20 & Milk, meat, eggs & $\mathrm{E}$ & $5.34 \mathrm{E}+13$ & $\mathrm{~J} /$ year & $2.58 \mathrm{E}+05$ & 137.73 \\
\hline 21 & Wood & $\mathrm{E}$ & $1.47 \mathrm{E}+14$ & $\mathrm{~J} /$ year & $5.04 \mathrm{E}+04$ & 74.31 \\
\hline 22 & Waste & $\mathrm{E}$ & $1.93 \mathrm{E}+13$ & $J /$ year & $9.59 \mathrm{E}+04$ & 18.47 \\
\hline 23 & Vehicles/scrap & $\mathrm{E}$ & $3.33 \mathrm{E}+08$ & $\mathrm{~g} /$ year & $6.28 \mathrm{E}+09$ & 20.92 \\
\hline 24 & Wastewater & $\mathrm{E}$ & $1.51 \mathrm{E}+12$ & $\mathrm{~m}^{3} /$ year & $3.80 \mathrm{E}+06$ & 57.54 \\
\hline
\end{tabular}


Notes to table 1

1 Solar energy

Global radiation Jokioinen $=3.32 \mathrm{E}+9 \mathrm{~J} / \mathrm{m}^{2}$ yr (Finnish Meteorological Institute 25.5.2009

http://www.fmi.fi/saa/tilastot_9.html\#6), Humpilan area $=1.48 \mathrm{E}+8 \mathrm{~m}^{2}$ of which water $=6.3 \mathrm{E}+5 \mathrm{~m}^{2}$ agricultural area $=5.91 \mathrm{E}+7 \mathrm{~m}^{2}$, forest area $=6.7 \mathrm{E}+7 \mathrm{~m}^{2}$, other area $=2.15 \mathrm{E}+7 \mathrm{~m}^{2}$ (Sinkko 2009), albedo: water area $=5 \%$, agricultural area $(15+18) / 2=16.5 \%$, forest area $(11+13) / 2=12 \%$. Other area $(15+16) / 2=$ $15.5 \%$ (estimated from Venäläinen et al. 1998).

Solar energy received $=3.32 \mathrm{E}+9 \mathrm{~J} / \mathrm{m}^{2} \mathrm{yr} *\left[\left(6.3 \mathrm{E}+5 \mathrm{~m}^{2} *(1-0.05)+5.91 \mathrm{E}+7 \mathrm{~m}^{2} *(1-0.165)+6.7 \mathrm{E}+7\right.\right.$ $\left.\mathrm{m}^{2} *(1-0.12)+2.15 \mathrm{E}+7 *(1-0.155)\right]=4.22 \mathrm{E}+17 \mathrm{~J} / \mathrm{yr}$.

Transformity: $1 \mathrm{sej} / \mathrm{J}$ per definition.

2 Wind kinetic energy

Energy $=$ Humpilan area $1.48 \mathrm{E}+8 \mathrm{~m}^{2} * 8.73 \mathrm{E}+10 \mathrm{~J} / \mathrm{ha}($ Franzese et al. 2009 , table 2$)=1.29 \mathrm{E}+13 \mathrm{~J}$.

Transformity $=2.51 \mathrm{E}+3 \mathrm{sej} / \mathrm{J}$ (Franzese et al. 2009 after Odum 1996).

3 Rain, evapotranspired

Energy $=$ Humpilan area $1.48 \mathrm{E}+8 \mathrm{~m}^{2} * 1.95 \mathrm{E}+10 \mathrm{~J} / \mathrm{ha}($ Franzese et al. 2009 , table 2$)=2.89 \mathrm{E}+12 \mathrm{~J} / \mathrm{yr}$.

Transformity $=3.05 \mathrm{E}+4 \mathrm{sej} / \mathrm{J}$ (Franzese et al. 2009 after Odum 1996).

4 Crop production

Data for crop yield of Humppila from (Information Centre of the Ministry of Agriculture and Forestry Finland 2007)

Energy $=82 \mathrm{E}+8 \mathrm{~g}$ cereals straw $* 17000 \mathrm{~J} / \mathrm{g}$ (estimated $)+(15 \mathrm{E}+8 \mathrm{~g}$ green fodder $+5 \mathrm{E}+8 \mathrm{~g}$ hay + $49 \mathrm{E}+8 \mathrm{~g}$ silage $+25 \mathrm{E}+8 \mathrm{~g}$ green fallow $+15 \mathrm{E}+8$ fallow $) * 16300 \mathrm{~J} / \mathrm{g}$ (estimated after Lampinen 2006) + $9 \mathrm{E}+8 \mathrm{~g}$ turnip rape straw $* 18700 \mathrm{~J} / \mathrm{g}$ (Lampinen 2006) $+(11 \mathrm{E}+6 \mathrm{~g}$ garden $+30 \mathrm{E}+6 \mathrm{~g}$ other $) * 3820 \mathrm{~J} / \mathrm{g}$ $($ estimated $)=3.34 \mathrm{E}+14 \mathrm{~J} / \mathrm{yr}+2.43 \mathrm{E}+14 \mathrm{~J} / \mathrm{yr}$ exported crop products $($ note 19$)=5.77 \mathrm{E}+14 \mathrm{~J} / \mathrm{yr}$.

Transformity: 3.31E+4 sej/J (after Rydberg et al. 2006, table A3).

5 Livestock production

Data for number of animals in Humppila from (Information Centre of the Ministry of Agriculture and Forestry Finland 2007). Data of number of animals and quantity of animal products in Finland from Statistical Yearbook of Finland 2004. Animal produce in 2003 divided by number of animals in Finland was used as mean value. Energy data from Rydberg et al. 2006 after Holland et al. 1993.

Energy $=95769 \mathrm{~g}$ beef/cattle $* 558$ cattle $* 10500 \mathrm{~J} / \mathrm{g}+6958140 \mathrm{~g}$ milk/cow $* 161$ cows $* 2760 \mathrm{~J} / \mathrm{g}+$ $320 \mathrm{~g} /$ veal $* 214$ veal $* 10500 \mathrm{~J} / \mathrm{g}+140530 \mathrm{~g} / \mathrm{pig} * 6311 \mathrm{pigs} * 15900 \mathrm{~J} / \mathrm{g}+21210 \mathrm{~g} /$ poultry $* 125438$ poultry $* 9630 \mathrm{~J} / \mathrm{g}+18630 \mathrm{~kg}$ eggs/hen $* 86828 \mathrm{hens} * 6150 \mathrm{~J} / \mathrm{g}+9640 \mathrm{~g}$ meat/horse $* 58$ horses $* 10500$ $\mathrm{J} / \mathrm{g}=5.34 \mathrm{E}+13 \mathrm{~J}$.

Transformity: $2.58 \mathrm{E}+5$ sej/J (Rydberg et al. 2006, table A3).

6 Forest extraction

Energy $=\left(4467 \mathrm{~m}^{3}\right.$ wood chips $+1250 \mathrm{~m}^{3}$ stumps $+2358 \mathrm{~m}^{3}$ energy wood $+15 \mathrm{E}+3 \mathrm{~m}^{3}$ round wood) $($ Sinkko $2008) * 53 \mathrm{E}+4 \mathrm{~g} / \mathrm{m}^{3} * 80 \%$ total solids $* 15069.6 \mathrm{~J} / \mathrm{g}$ (Rydberg et al. 2006 after Tsoumis 1991) $=1.47 \mathrm{E}+14$ $\mathrm{J} / \mathrm{yr}$.

Transformity: 5.04E+4 sej/J (Brown et al. 2007 after Brown 2001).

7 District Heating

Energy $=13000 \mathrm{MWh} / \mathrm{yr}($ Sinkko 2008, s 47) $* 3.6 \mathrm{E}+9 \mathrm{~J} / \mathrm{MWh}=4.68 \mathrm{E}+13 \mathrm{~J} / \mathrm{yr}$.

Transformity: $46 \mathrm{E}+12$ sej diesel $+7 \mathrm{E}+12$ sej steel farm machinery $+2.29 \mathrm{E}+14$ sej goods and services farm $+1 \mathrm{E}+12$ sej steel power plant $+1.68 \mathrm{E}+14$ sej electricity $+6.68 \mathrm{E}+14$ sej goods and services power plant $+5.1 \mathrm{E}+13$ sej goods and services ash $) / 3.52 \mathrm{E}+10 \mathrm{~J}$ heat $($ Nilsson 1997, table 3, p. 70) $=3.32 \mathrm{E}+4 \mathrm{sej} / \mathrm{J}$.

8 Top soil

Energy $=183 \mathrm{~g} / \mathrm{m}^{2} / \mathrm{yr}$ erosion rate $* 587 \mathrm{~J} / \mathrm{g} * 10000 \mathrm{~m}^{2} / \mathrm{ha}=1.08 \mathrm{E}+9 \mathrm{~J} / \mathrm{ha} \mathrm{yr}$ (Rydberg et al. 2006, B3 p. 21)

* 5909 ha agricultural area of Humppila (Information Centre of the Ministry of Agriculture and Forestry

Finland 2008) $=6.35 \mathrm{E}+12 \mathrm{~J} / \mathrm{yr}$.

Transformity: $1.24 \mathrm{E}+5 \mathrm{sej} / \mathrm{J}$ (Franzese et al. 2009, table 2).

9 Oil derived products

The energy of traffic fuel is calculated from the average of diesel and gasoline consumption in Finland (LIISA 2007): 168 PJ/yr Finland's traffic fuel / 12318480 t CO $/$ yr * 14300 t CO $/ \mathrm{yr} / 4559 \mathrm{t}$ fuel $\left(\mathrm{CO}_{2}\right.$ emission and fuel consumption of Humppila, Sinkko 2009, p. 50 and 43) $=4.28 \mathrm{E}+10 \mathrm{~J} / \mathrm{t}$ fuel.

Energy $=(15600 \mathrm{MWh}$ detached house heating $* 30 \%$ oil (Sinkko 2009, p. 48) $+11220 \mathrm{MWh}$ oil grain drying (Sinkko 2009, s 47)) * 3.6E+9 J/MWh) $+800 \mathrm{tCO}_{2}$ (Sinkko 2009, p. 49) * 1000kg/t* $830 \mathrm{~g} / \mathrm{l} \mathrm{diesel}$ farm machinery $/ 2.7 \mathrm{~kg} \mathrm{CO} / 1 / 1000000 \mathrm{~g} / \mathrm{t} * 4.28 \mathrm{E}+10 \mathrm{~J} / \mathrm{t}$ fuel $)+4559 \mathrm{t}$ Humpilan traffic fuel, diesel and gasoline * 4.28E+10 J/g* $1 \mathrm{E}+6 \mathrm{~g} / \mathrm{t}=2.63 \mathrm{E}+14 \mathrm{~J} / \mathrm{yr}$.

Transformity: 1.11E+5 sej/J (Franzese 2009, table 2).

10 Electric power

Energy $=22600 \mathrm{MWh} / \mathrm{yr}($ Sinkko 2009, p. 46) $* 3.6 \mathrm{E}+9 \mathrm{~J} / \mathrm{MWh}=8.14 \mathrm{E}+13 \mathrm{~J} / \mathrm{yr}$. 
Transformity: 2.51E+5 sej/J (Franzese 2009, table 1 after Brown et al. 2004).

11 Metals

Energy $=20 \mathrm{t}$ metal scarp/yr (Sinkko 2009, p. 45) $* 1 \mathrm{E}+6 \mathrm{~g} / \mathrm{t}=2 \mathrm{E}+7 \mathrm{~g} / \mathrm{yr}$.

12 Nitrogen

Transformity: 6.28E+9 sej/g (Buranarkan 1989, p. 206).

Mass $=80 \mathrm{~kg} \mathrm{~N} /$ ha (Statistics Finland 2004) $* 5909$ ha (Information Centre of the Ministry of Agriculture and Forestry Finland 2008) $* 1000 \mathrm{~g} / \mathrm{kg}=\mathrm{t} 4.73 \mathrm{E}+8 \mathrm{~g} / \mathrm{yr}$.

13 Phosphate

Transformity: 7.71E+9 sej/g (Franzese 2009, table 2 after Odum 1996).

Mass $=10 \mathrm{~kg}$ P/ha (Statistics Finland 2004) * 5909 ha (Information Centre of the Ministry of Agriculture and Forestry Finland 2008) $* 1000 \mathrm{~g} / \mathrm{kg}=5.91 \mathrm{E}+7 \mathrm{~g} / \mathrm{yr}$.

14 Potash

Transformity: 2.98E+10 sej/g (Franzese 2009, table 2 after Odum 1996).

Mass $=28 \mathrm{~kg} \mathrm{~K} / \mathrm{ha}$ (Statistics Finland 2004) $* 5909$ ha (Information Centre of the Ministry of Agriculture and Forestry Finland 2008) $* 1000 \mathrm{~g} / \mathrm{kg}=1.65 \mathrm{E}+8 \mathrm{~g} / \mathrm{yr}$.

Transformity: 2.92E+9 sej/g (Franzese 2009, table 2 after Odum 1996).

15 Pesticides \& Herbicides

Mass $=5498 \mathrm{t} / 1965000$ ha (Statistics Finland 2004) * 5909ha (Information Centre of the Ministry of

Agriculture and Forestry Finland 2008) $* 1000 \mathrm{~g} / \mathrm{kg}=1.65 \mathrm{E}+7 \mathrm{~g} / \mathrm{yr}$

Transformity: 2.48E+10 sej/g (Franzese 2009, table 2 after Brawn et al. 1991).

16 Feed

Import cost $=1.23 \mathrm{E}+8 €$ Prepared animal fodder $/(1.93 \mathrm{E}+8 \mathrm{~kg}$ pork $+8.37 \mathrm{E}+7 \mathrm{~kg}$ poultry $)($ feed import and meat production Finland, Statistical Yearbook of Finland 2004, 230/23)* $(8.87 \mathrm{E}+5 \mathrm{~kg}$ pork $+2.66 \mathrm{E}+6 \mathrm{~kg}$ poultry) (meat production Humppila, Information Centre of the Ministry of Agriculture and Forestry Finland $2008)=1.58 \mathrm{E}+6 € / \mathrm{yr}$.

Transformity: National Emergy Money Ratio (EMR) 4.04E+12 sej/ $\$ 1.19 \$ / €=3.39 \mathrm{E}+12 \mathrm{sej} / €$ (Cohen et al. 2009).

17 Vehicles

Calculation: number of vehicles (Sinkko 2009, s 43)* weight/vehicle (estimated) * life time (estimated) * 206.3MJ/kg (Gaillard et al. 1997, p24 class A1)

Energy $=(1451 \mathrm{cars} * 1500 \mathrm{~kg} / \mathrm{car} / 20 \mathrm{yr}+349$ lorries and buses $* 5000 \mathrm{~kg} / \mathrm{lorry}$ and bus $/ 20 \mathrm{yr}+141$ motorbikes $* 300 \mathrm{~kg} /$ motorbike $/ 10 \mathrm{yr}+114$ soccer $* 150 \mathrm{~kg} /$ soccer $/ 10 \mathrm{yr}+28 \mathrm{rakes} * 150 \mathrm{~kg} / \mathrm{rake} / 10 \mathrm{yr}$ +558 tractors $* 3000 \mathrm{~kg} /$ tractor $/ 20 \mathrm{yr}+94$ working machinery $* 5000 \mathrm{~kg} /$ working machine $/ 10 \mathrm{yr})=$ $3.33 \mathrm{E}+8 \mathrm{~g} / \mathrm{yr}$.

Transformity: 6.7E+9 sej/g (Odum et al. 1983)

18 Service and food imports

The total of Humppila's emergy is calculated by multiplying the national emergy money ratio with consumption of Finnish households and with the ratio between Humppila's and national households and subtracting the total of imported goods and services marked by note 9 to 17 to avoid double counting:

Emergy $=3.39 \mathrm{E}+12 \mathrm{sej} / €($ Cohen et al. 2009) $* 66657 \mathrm{E}+6 €$ national consumption (Statistics Finland $2004) * 1179$ households in Humppila (Sinkko 2009) / 2378079 households in Finland (Statistics Finland $2004)-6.36 \mathrm{E}+19$ sej (total of note 9 to 17$)=4.86 \mathrm{E}+19$ sej.

The services and goods imported to Humppila: $4.86 \mathrm{E}+19 \mathrm{sej} / 3.39 \mathrm{E}+12 \mathrm{sej} / €=1.43 \mathrm{E}+7 €$.

19 Exported crop products

Crop yield of Humppila from Information Centre of the Ministry of Agriculture and Forestry Finland 2007.

Energy $=131 \mathrm{E}+8 \mathrm{~g}$ cereals $* 17000 \mathrm{~J} / \mathrm{g}($ Lampinen 2006$)+7 \mathrm{E}+8 \mathrm{~g}$ turnip rape $* 26400 \mathrm{~J} / \mathrm{g}$ (Lampinen $2006)+77 \mathrm{E}+6 \mathrm{~g}$ peas $* 3470 \mathrm{~J} / \mathrm{g}$ (Rydberg et al. 2006 after Holland et al. 1993) $+522 \mathrm{E}+6 \mathrm{~g}$ potatoes $* 3350$ $\mathrm{J} / \mathrm{g}$ (estimated) $+33 \mathrm{E}+6 \mathrm{~g}$ horticulture products $* 3820 \mathrm{~J} / \mathrm{g}$ (estimated) $=2.34 \mathrm{E}+14 \mathrm{~J} / \mathrm{yr}$.

Transformity: 1.59E+5 sej/J (Rydberg et al., 2006, table A3).

20 Exported milk, meat, eggs

See note 5.

21 Exported wood

See note 6.

22 Exported waste

Mass and total solids (TS) after Sinkko 2009, transformities after Buranarkan1989, p. 206.

Energy $=77 \mathrm{E}+7 \mathrm{~g}$ municipal solid waste $* 71.5 \% \mathrm{TS} * 21500 \mathrm{~J} / \mathrm{g}+21 \mathrm{E}+7 \mathrm{~g}$ paper and paperboard $*$

$93.6 \% \mathrm{TS} * 19600 \mathrm{~J} / \mathrm{g}+1 \mathrm{E}+8 \mathrm{~g}$ food $* 33 \% \mathrm{TS} * 5 \mathrm{kcal} / \mathrm{g} * 4186 \mathrm{~J} / \mathrm{kcal}$ (Buranarkan 1989, p. 208) $+7 \mathrm{E}+7$ $\mathrm{g}$ glass $*(1.83 \mathrm{E}+16 \mathrm{~J}$ fuel $+5.95 \mathrm{E}+15 \mathrm{~J}$ electricity) $/ 3.03 \mathrm{E}+12 \mathrm{~g}$ flat glass with services (Buranarkan 1989, p. 195) $+2 \mathrm{E}+7 \mathrm{~g}$ metal $* 696 \mathrm{~J} / \mathrm{g}($ Buranarkan 1989, p. 155) $+2 \mathrm{E}+7 \mathrm{~g}$ wood $* 75 \% \mathrm{TS} * 19100 \mathrm{~J} / \mathrm{g}($ Rydberg 
et al. 2006 after Tsoumis, 1991) + 1E+7 g plastics * $31800 \mathrm{~J} / \mathrm{g} /$ Sinkko 2009) * 2E+7 g electronics * 33000 $\mathrm{J} / \mathrm{g}($ estimated $)+5 \mathrm{E}+7 \mathrm{~g}$ other $* 20900 \mathrm{~J} / \mathrm{g}$ (estimated) $=193 \mathrm{E}+11 \mathrm{~J} / \mathrm{yr}$.

Emergy $=77 \mathrm{E}+7 \mathrm{~g}$ municipal solid waste $* 71.5 \% \mathrm{TS} * 279 \mathrm{E}+7 \mathrm{sej} / \mathrm{g}+21 \mathrm{E}+7 \mathrm{~g}$ paper and paperboard $* 93.6 \% \mathrm{TS} * 142 \mathrm{E}+3 \mathrm{sej} / \mathrm{g}+1 \mathrm{E}+8 \mathrm{~g}$ food $* 33 \% \mathrm{TS} * 1 \mathrm{E}+5 \mathrm{sej} / \mathrm{g}+7 \mathrm{E}+7 \mathrm{~g}$ glass $* 19 \mathrm{E}+8 \mathrm{sej} / \mathrm{g}+2 \mathrm{E}+7 \mathrm{~g}$ metal $* 628 \mathrm{E}+7 \mathrm{sej} / \mathrm{g}+2 \mathrm{E}+7 \mathrm{~g}$ wood $* 75 \% \mathrm{TS} * 8010 \mathrm{sej} / \mathrm{g}+1 \mathrm{E}+7 \mathrm{~g}$ plastics $* 328 \mathrm{E}+8 \mathrm{sej} / \mathrm{g}+2 \mathrm{E}+7 \mathrm{~g}$ electronics $* 1 \mathrm{E}+9 \mathrm{sej} / \mathrm{g}+5 \mathrm{E}+7 \mathrm{~g}$ other $* 8010 \mathrm{sej} / \mathrm{g}=1.85 \mathrm{E}+18 \mathrm{sej}$.

Transformity $=1.85 \mathrm{E}+18 \mathrm{sej}($ Emergy $) / 193 \mathrm{E}+11 \mathrm{~J}($ Energy $)=9.59 \mathrm{E}+4 \mathrm{sej} / \mathrm{g}$.

23 Vehicles/scrap

Emergy $=3.33 \mathrm{E}+8 \mathrm{~g} / \mathrm{yr}($ note 22$) * 6.28 \mathrm{E}+9 \mathrm{sej} / \mathrm{g}($ metal, Buranarkan 1989, p. 206) $=2.09 \mathrm{E}+18 \mathrm{sej} / \mathrm{yr}$. 24 Wastewater

Energy $=200000 \mathrm{~m}^{3} / \mathrm{a}\left(\right.$ Sinkko 2009, s 46) $* 7.57 \mathrm{E}+6 \mathrm{~J} / \mathrm{m}^{3}$ (Björklund 2001, p. 309) = 1.51E+12 J/yr.

Transformity: 3.8E+6 sej/J (Björklund 2001, p. 300).

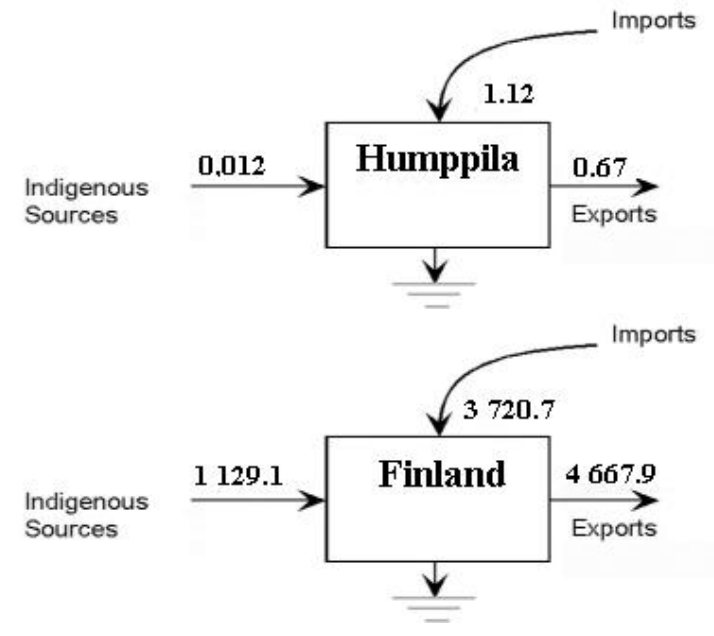

Figure 2: Summary diagram of resource bases of Humppila (this work) and Finland. Unit E+20 sej/year. The indigenous sources are the sum of renewable (solar radiation in Humppila, total water in Finland (Cohen et al. 2009) and non-renewable sources. Only the highest value of renewable sources is accounted for to avoid double counting (Odum 1996). The other renewable sources result from solar radiation too.

The total emergy $\mathrm{Y}=\mathrm{R}+\mathrm{N}+\mathrm{F}+\mathrm{G}+\mathrm{S}$ used in Humppila is $1.13 \mathrm{E}+20$ sej. Goods and services $(4.85 \mathrm{E}+19 \mathrm{sej})$, oil derived products $(2.92 \mathrm{E}+19 \mathrm{sej})$, and electric power $(2 \mathrm{E}+19 \mathrm{sej})$ reach $46 \%$ of the imports. The total renewable emergy is approximately $0.4 \%$ of the total used emergy. This is because near all food and goods come from outside. Therefore, the environmental load ratio $\mathrm{ELR}=(\mathrm{Y}-\mathrm{R}) / \mathrm{R}$, that gives a measure of the ability to use local resources, is extremely high. It amounts to 268 in Humppila compared to 23.3 in Finland. Accordingly the emergy investment ratio $\mathrm{EIR}=(\mathrm{F}+\mathrm{G}+\mathrm{S}) /(\mathrm{R}+\mathrm{N})$, i.e. the emergy investment compared to the emergy of local resource use, shows that the environmental and economic system of Humppila is not very sustainable because of its dependence on inputs from outside economic systems. The ratio of Humppila is 93 compared to 3.3 of Finland.

\section{Conclusion}

Although Humppila has a good potential to replace fossil fuels by renewable energy sources, the environmental and economic system is very vulnerable. Beside the question, which technology grants the highest energy return on investment in terms of Joule/Joule to produce heat and power from local renewable resources, another question must be answered: How to reduce the indirect dependency from fossil fuels, hidden in goods and services from outside. Local food processing an extended use of renewable natural raw materials may be the most promising way. 


\begin{abstract}
References
Bastianoni, S. Pulselli, R., Pulselli, F. 2009. Models of withdrawing renewable and non-renewable resources based on Odum's energy systems theory and Daly's quasi-sustainability principle. Ecol. Model. 220: 1926-1930. Björklund, J., Geber, U., Rydberg, T. 2001. Emergy analysis of municipal wastewater treatment and generation of electricity by digestion of sewage sludge. Resources, Conservation and Recycling 31: 293-316. Brown, M., Arding, J. 1991. Transformities working paper. Center for Environmental Policy, University of Florida, Gainesville.
\end{abstract}

Brown, M., Bardi, E. 2001. Handbook of Emergy Evaluation Folio \#3. Center for Environmental Policy, University of Florida, Gainesville, Fl.30 pp.

Brown, M., Campbell, E. 2007. Evaluation of natural capital and environmental services of U.S. national forests using emergy synthesis. Final report. Center for environmental policy University of Florida. PO Box 116350, Gainesville, FL 32611.

Brown, M., Ulgiati, S., 2004. Emergy analysis and environmental accounting. In: Cleveland, C. (Ed.), Encyclopedia of Energy. Academic Press, Elsevier, Oxford, UK, pp. 329-354.

Buranakarn, V., 1998. Evaluation of recycling and reuse of building materials using the emergy analysis method. Ph.D. Dissertation. University of Florida. 279 p.

Cohen, M., Brown, M., Sweeney, S., King, D. 2009. Environmental Accounting and Systems Synthesis of Land Management Interventions at Multiple Scales in the Sahel Region of West Africa University of Florida. http://sahel.ees.ufl.edu/database_resources.php?search_type=basic\&country=FIN (24.11.09).

Franzese, P., Rydberg, T., Russo, G., Ulgiati, S. 2009. Sustainable biomass production: A comparison between Gross Energy Requirement and Emergy Synthesis methods. Ecological indicators 9: 959-970.

Gaillard, G., Crettaz, P., Hausheer, J. 1997 Umweltinventar der landwirtschaftlichen Inputs im Pflanzenbau. Eidgenössische Forschungsanstalt für Agrarwirtschaft und Landtechnik, CH-8356 Tänikon TG, 45 p.

Heinimö, J. 2008. IEA BIOENERGY TASK 40. ”Sustainable International Bioenergy Trade: Securing supply and demand". Country report of Finland 2008. Research report EN A-57. ISBN 978-952-214-613-7. 32 p.

Holland, B., Welch, A., Unwin I.,, Buss, D., Paul, A., Southgate, D. 1993. McCance \& Widdowson's. The Composition of Foods. Royal Society of Chemistry and Ministry of Agriculture, Fisheries and Food. 5th ed., Cambridge.

Jiang, M., Chen, B., Zhou, J. Tao, F., Li, Z., Yang, Z. Chen, G. 2007. Emergy account for biomass resource exploitation by agriculture in China. Energy Policy 35: 4704-4719.

Information Centre of the Ministry of Agriculture and Forestry Finland. www.mmmtike.fi/en/index.html http://www.matilda.fi/servlet/page?_pageid=115,193\&_dad=portal30\&_schema=PORTAL30. Access 24.11.09. Lampinen, A., Jokinen, E. 2006. Suomen maatilojen energiatuotantopotentiaalit. University of Jyväskylä, bioja ympäristötieteiden laitos. Research reports in biological and environmental sciences 84:1-160.

LIISA, 2007. Suomen tieliikenteen pakokaasupäästöjen laskentajärjestelmä.http://lipasto.vtt.fi/liisa/index.htm (accessed 12.12.2008).

Odum, H. 1996: Environmental Accounting, Emergy and Decision Making. John Wiley, NY, 370 p.

Odum, H., Odum, E. eds. 1983. Energy Analysis Overview of Nations. Working Paper WP-83-82.

International Institute for Applied Systems Analysis, Laxenburg, Austria. 469 p.

Paoli, C., Vassallo, P., Fabiano, M. 2008. Solar power: An approach to transformity evaluation. Ecological Engineering 34: 191-206.

Pulselli, R., Pulselli, F., Rustici, M. 2008. Emergy accounting of the Province of Siena: Towards a thermodynamic geography for regional studies. Journal of Environmental Management 86: 342-353.

Rydberg, T., Haden, A. 2006. Emergy evaluations of Denmark and Danish agriculture: Assessing the influence of changing resource availability on the organization of agriculture and society. Agriculture, Ecosystems and Environment 117: 145-158.

Schäfer, W. 2008. Energy crops and renewable energy: overall and process efficiency. In: Toim. Anneli Hopponen. Maataloustieteen Päivät 2008, 10.-11.1.2008. Suomen maataloustieteellisen seuran tiedote 23: 6 p. http://www.smts.fi/mpol2008/index_tiedostot/Esitelmat/es037.pdf

SFS-EN ISO 14040 2006. Life cycle assessment. Principles and framework 49 p.

Siracusa, G., La Rosa, A., Palma, P., La Mola, E. 2008. New frontiers for sustainability: emergy evaluation of an eco-village. Environment, Development and Sustainability 10: 845-855.

Sinkko, T. 2009. Pienen mittakaavan CHP-laitokset osana hiilineutraalia maaseutuyhteiskuntaa. Diplomityö Lappeenrannan teknillinen yliopisto Teknillinen tiedekunta. $94 \mathrm{p}$.

Statistics Finland 2004. Statistical Yearbook of Finland 99: 718 p.

Tsoumis, G. 1991. Science and Technology of Wood: Structure, properties, Utilization. Van Nostrand Reinhold, New York 494 p.

Venäläinen, A, Solantie, R., Laine, V. 1998. Mean long-term surface energy balance components in Finland during summertime. Boreal Environment Research 3: 171-180. 